\title{
Saturation of transgene protein synthesis from mRNA in cells producing a large number of transgene mRNA.
}

\section{AUTHOR(S):}

Takahashi, Yuki; Nishikawa, Makiya; Takiguchi, Naomi; Suehara, Tetsuya; Takakura, Yoshinobu

\section{CITATION:}

Takahashi, Yuki ... [et al]. Saturation of transgene protein synthesis from mRNA in cells producing a large number of transgene mRNA.. Biotechnology and bioengineering 2011, 108(10): 2380-2389

\section{ISSUE DATE:}

2011-05-02

URL:

http://hdl.handle.net/2433/197140

\section{RIGHT:}

This is the peer reviewed version of the following article: Takahashi, Y., Nishikawa, M., Takiguchi, N., Suehara, T. and Takakura, Y. (2011), Saturation of transgene protein synthesis from mRNA in cells producing a large number of transgene mRNA. Biotechnol. Bioeng., 108: 2380-2389, which has been published in final form at http://dx.doi.org/10.1002/bit.23179; この論文は出版社版でありません。引用の際には出版社版をご確認ご利用くださ $\iota_{0}$; This is not the published version. Please cite only the published version. 


\section{Saturation of transgene protein synthesis from mRNA in cells producing a large number of transgene mRNA}

Yuki Takahashi, Makiya Nishikawa, Naomi Takiguchi, Tetsuya Suehara and Yoshinobu Takakura Department of Biopharmaceutics and Drug Metabolism,

Graduate School of Pharmaceutical Sciences, Kyoto University, Kyoto, 606-8501, Japan

Address correspondence to: Makiya Nishikawa PhD, 46-29 Yoshidashimoadachi-cho, Sakyo-ku, Kyoto 606-8501, Japan. Phone: 81-75-753-4580; Fax: 81-75-753-4614; E-mail:

makiya@pharm.kyoto-u.ac.jp

Running title: Saturation in transgene expression processes 


\begin{abstract}
Experimental results have suggested that transgene expression can be saturated when large amounts of plasmid vectors are delivered into cells. To investigate this saturation kinetic behavior, cells were transfected with monitoring and competing plasmids using cationic liposomes. Even although an identical amount of a monitoring plasmid expressing firefly luciferase was used for transfection, transgene expression from the plasmid was greatly affected by the level of transgene expression from competing plasmids expressing renilla luciferase. Similar results were obtained by exchanging the monitoring and competing plasmids. The competing plasmid-dependent reduction in transgene expression from the monitoring plasmid was also observed in mouse liver after hydrodynamic injection of plasmids. On the other hand, the mRNA and protein expression level of GAPDH, an endogenous gene, in the liver hardly changed even when transgene expression process is saturated. The expression of firefly luciferase from a monitoring plasmid was significantly restored by siRNA-mediated degradation of renilla luciferase mRNA that was expressed from a competing plasmid. These results suggest that the efficiency of protein synthesis from plasmid vectors is reduced when a large amount of mRNA is transcribed with no significant changes in endogenous gene expression.
\end{abstract}

Key Words: Saturation, Transgene expression, Plasmid vector, mRNA expression, RNA interference. 


\section{INTRODUCTION}

Development of efficient gene delivery methods is needed not only for gene therapy but also for the functional studies of genes. Of the various vectors and gene delivery/transfer methods developed thus far, rapid injection of naked plasmid DNA in a large volume of isotonic solution, the so-called the hydrodynamic injection method, is one of the most effective and promising gene delivery methods because of its simplicity, reproducibility and high efficiency (Herweijer and Wolff 2007; Kobayashi et al. 2005; Lewis and Wolff 2007; Liu et al. 1999). Therefore, this gene delivery technique has frequently been used as an experimental tool to investigate the gene of interest in mouse liver, the organ that most efficiently expresses the gene products after hydrodynamic delivery of naked plasmid DNA.

In a previous study, we found, using a firefly luciferase-expressing plasmid DNA, that the level of luciferase activity in mouse liver increased in parallel with the increasing dose of the plasmid up to about $1 \mu \mathrm{g}$ DNA/mouse (Kobayashi et al. 2004). However, we also noticed that the luciferase activity reached a plateau at doses around $10 \mu \mathrm{g}$ DNA/mouse, suggesting that the transgene expression from plasmid DNA is saturated at such high doses. These experimental results could be explained by assuming the presence of one or more saturable processes in the transgene expression after hydrodynamic delivery of plasmid DNA to mouse liver. One obvious possibility is that the amount of plasmid DNA delivered to the nucleus of transfected cells is not proportional to the increasing dose of the DNA. However, this can be rejected by the previous 
finding that $20 \mu \mathrm{g}$ empty plasmid co-administered had no significant effect on the level of transgene expression from $0.2 \mu \mathrm{g}$ luciferase-expressing plasmid (Kobayashi et al. 2004). The empty plasmid used contained the same promoter/enhancer and other components as those in the luciferase-expressing plasmid except for the cDNA region. Therefore, these results strongly support the hypothesis that the efficiency of the cellular uptake and intracellular trafficking of plasmid DNA, including the nuclear entry, is not a function of the amount or concentration of the DNA, even although several reports have indicated that transcription factors and other proteins were involved in the nuclear entry of plasmid DNA microinjected into the cytosol (Dean 1997; Dean et al. 1999; Miller and Dean 2008).

There are several steps in the process of transgene expression, including RNA synthesis (transcription), RNA modification (splicing), export of mRNA from the nucleus into the cytosol, protein synthesis (translation), post-translational modification of protein and transport/trafficking of protein. Considering the fact that all these steps in the gene expression are capacity-limited, it is reasonable that transgene expression becomes saturated when a large amount of DNA is introduced into cells. In good agreement with this hypothesis, saturation in transgene expression has been discussed in several studies involving transgene expression in cultured cells. Tachibana et al. investigated the relationship between the dose of plasmid DNA added to cells and the amount of transgene product (Tachibana et al. 2002). They showed that the copy number of plasmid DNA in the nucleus correlated with its dose. Furthermore, they demonstrated that the 
copy number in the nucleus did not correlate with the amount of transgene product, especially when the copy number was high. A recent study by Cohen et al. also showed that nuclear uptake of plasmid DNA was linearly related to its dose whereas the level of transgene expression was not (Cohen et al. 2009). Carpentier et al. investigated the limiting factors involved in the process of transgene expression (Carpentier et al. 2007). They found that both transcriptional and translational processes were saturated under optimal transfection conditions in which quite a high level of gene expression was obtained. These previous studies strongly suggest that transgene expression can be saturated under certain conditions.

Detailed understanding of this saturable mechanism of transgene expression from plasmid vectors will provide new insights into how gene expression is regulated in cells. The understanding could also lead to the development of methods to achieve high level of transgene expression without saturation. In addition, from the viewpoint of safety, it is important to investigate whether endogenous gene expression is affected when transgene expression process is saturated, because it may cause unexpected side effects, such as cell death. For example, Lin et al. reported that adenoviral vector-mediated high transgene expression of serum response factor resulted in nonspecific reduction of transcription of endogenous genes and caused cell death (Lin et al. 2007). Therefore, in the present study, we examined whether the synthesis of transgene products is actually saturated when a large amount of plasmid DNA is delivered to cells. To this end, cultured cells were transfected with two types of plasmid vectors, each of which encodes a 
reporter protein, and transgene expression from the vectors was used to investigate whether the expression from one plasmid (monitoring plasmid) is affected by that from the other plasmid (competing plasmid). Then, similar experiments were carried out using mice that received a hydrodynamic injection of the two plasmid vectors. In addition, the mRNA and protein levels of glyceraldehyde-3-phosphate dehydrogenase (GAPDH) in mouse liver were evaluated as a model endogenous gene to evaluate the effect of transgene expression on endogenous gene expression. Small interfering RNA (siRNA) was used to reduce the amount of mRNA without altering the amount of plasmids for transfection.

\section{MATERIALS AND METHODS}

\section{Plasmid DNA and siRNA}

Salmon testes DNA (stDNA) was purchased from Sigma (St Louis, MO, USA). Plasmid DNA encoding firefly luciferase (FL) under the control of CMV promoter (pFL-CMV) was constructed as described previously (Nomura et al. 1999). The following plasmids were purchased from the sources in brackets: pGL3-control vector (Promega, Madison, WI, USA), pLuc-mcs (Stratagene, La Jolla, CA, USA), pRL-CMV (Promega) encoding renilla luciferase (RL) under the control of CMV promoter, pRL-SV40 (Promega), pRL-TK (Promega), pEGFP-F (BD Biosciences Clontech, Palo Alto, CA, USA) encoding farnesylated enhanced green 
fluorescent protein (GFP) under the control of CMV promoter. These plasmids were renamed according to the cDNA and promoter of each plasmid as follows: pFL-SV40, pFL-TATA, pRL-CMV, pRL-SV40, pRL-TK and pGFP-CMV. siRNA targeting renilla luciferase mRNA (siRL) or GFP mRNA (siGFP) was purchased from Takara Bio (Otsu, Japan). Target sites in the renilla luciferase and GFP mRNA are as follows: renilla luciferase, 5'-GUAGCGCGGUGUAUUAUAC -3'; GFP, 5'-GGCUACGUCCAGGAGCGCA-3'.

\section{Cell culture}

A murine melanoma cell line, B16-BL6, was obtained from the Cancer Chemotherapy Center of the Japanese Foundation for Cancer Research (Poste et al. 1980). B16-BL6 cells were cultured in Dulbecco’s modified Eagle’s minimum essential medium (Nissui Pharmaceutical, Tokyo, Japan) supplemented with $10 \%$ fetal bovine serum and penicillin (100 U/ml)/streptomycin (100 $\mu \mathrm{g} / \mathrm{ml}) / \mathrm{L}$-glutamine $(2 \mathrm{mM})$ at $37{ }^{\circ} \mathrm{C}$ and $5 \% \mathrm{CO}_{2}$.

\section{In vitro transfection}

B16-BL6 cells were plated on 24-well culture plates at the density of $2 \times 10^{4}$ cells/well. After an overnight incubation, transfection of plasmid DNA was carried out using Lipofectamine 2000 (Invitrogen, Carlsbad, CA, USA) according to the manufacturer's instructions. In brief, $1 \mu \mathrm{g}$ of nucleic acids (plasmids with or without siRNA), was mixed with $3 \mu$ g Lipofectamine 2000 in 
Opti-MEM I (Invitrogen) at a final concentration of $6 \mu$ g Lipofectamine 2000/ml. The resulting complex was added to cells and the cells were incubated with the complex for $4 \mathrm{hr}$. Then, cells were washed with PBS and further incubated with the culture medium.

\section{Luciferase assay in B16-BL6 cells}

B16-BL6 cells were lysed using the cell lysis buffer of an assay kit (PiccageneDual, Toyo Ink, Tokyo, Japan). Then, samples were mixed with the kit luciferase assay buffer, and the chemiluminescence produced was measured in a luminometer (Lumat LB9507, EG and G Berthold, Bad Wildbad, Germany). The luciferase activities were converted to the amount of firefly and renilla luciferase using recombinant proteins as the standard.

\section{Flow cytometric analysis of GFP expression in B16-BL6 cells}

B16-BL6 cells were transfected with pGFP-CMV as described above. One day after transfection, adherent cells were detached by trypsinization and resuspended in PBS. Resuspended cells were analyzed on a flow cytometer (FACSCan, BD, Franklin Lakes, NJ, USA). The threshold on FSC was set to exclude cell debris without excluding any populations of interest.

\section{mRNA quantification in B16-BL6 cells}

One day after transfection, total RNA was extracted from cells using Sepasol RNA I super 
(Nacalai Tesque, Kyoto, Japan). Following RNase-free DNase I treatment (Takara Bio), reverse transcription was performed using a SuperScript II (Invitrogen) and dT-primer according to the manufacturer's protocol. For a quantitative analysis of mRNA expression, real-time PCR was carried out with total cDNA using a Light-Cycler instrument (Roche Diagnostics, Basle, Switzerland) as reported previously (Takahashi et al. 2005). The mRNA expression of target genes was normalized using the mRNA level of GAPDH.

\section{Animal experiments}

Four-week-old male ICR mice (approximately 20g body weight), purchased from Japan SLC, Inc. (Shizuoka, Japan) were used for all experiments. The protocols for animal experiments were approved by the Animal Experimentation Committee of the Graduate School of Pharmaceutical Sciences of Kyoto University. Mice received an intravenous injection of plasmid DNA by the hydrodynamic injection method as described previously (Liu et al. 1999). At 6 hr after gene transfer, the peak time of the expression of firefly and renilla luciferases from the plasmid vectors used, the mice were killed by cervical dislocation and the amount of luciferase mRNA and protein in the liver was determined as described below.

\section{Quantification of the amounts of luciferase and GAPDH in mouse liver}

The liver was excised and homogenized in a lysis buffer (0.1 M Tris (pH 7.8), 0.05\% Triton 
X-100, 2 mM EDTA, $0.1 \%$ protease inhibitor cocktail (Sigma)), and centrifuged at $13000 \mathrm{~g}$ for

$20 \mathrm{~min}$ at $4{ }^{\circ} \mathrm{C}$. The amount of luciferase in the supernatant was determined by the method described in the above section. The amount of GAPDH protein in the lysate was measured by ELISA by using a commercial kit (GAPDH Whole-cell Normalization kit, Active Motif, Carlsbad, CA).

\section{mRNA quantification in mouse liver}

Total RNA was extracted from approximately 50 mg liver samples using Sepasol RNA I Super. After purification of the extracted RNA sample using an RNeasy mini kit (Qiagen), reverse transcription and real-time PCR analysis were performed as described in the above section.

\section{Statistical analysis}

The normality of the data was evaluated by using Shapiro-Wilk test. Data of normal distribution were analyzed by Student's t-test for two independent samples or one-way analysis of variance (ANOVA) followed by the Tukey's test for multiple comparisons. Data without normal distribution were analyzed using Mann-Whitney rank sum test for two independent samples or Kruskal-Wallis one-way ANOVA on ranks followed by the Tukey’s test for multiple comparisons. A $P$-value of less than 0.05 was considered to be statistically significant. 


\section{RESULTS}

Time-course and dose-dependent luciferase expression in B16-BL6 cells.

Fig. 1A shows the time-courses of the amounts of firefly and renilla luciferases in cells after cotransfection with pFL-CMV and pRL-CMV. The expression of both luciferases was detectable as early as $6 \mathrm{hr}$ after transfection, reached maximum values at $24 \mathrm{hr}$ and then eventually decreased with time. As the highest luciferase expression was detected at $24 \mathrm{hr}$ after transfection under the conditions used, this time point was selected and used in the following experiments to examine transgene expression in B16-BL6 cells.

Fig. 1B shows the amount of firefly luciferase in cells transfected with different amounts of pFL-TATA, pFL-SV40 or pFL-CMV. pFL-CMV showed the highest firefly luciferase, followed by pFL-SV40, then pFL-TATA, reflecting the strength of these promoters used. When cells were transfected with one of the renilla luciferase-expressing plasmid DNA, pRL-CMV showed the highest renilla luciferase activity, followed by pRL-SV40 and pRL-TK (data not shown).

To statistically evaluate whether the expression is saturated with an increasing amount of plasmid DNA, the expression efficiency was estimated by dividing the luciferase activity by the dose (Fig. 1C). The value was about 40,000 pg/well/ $\mu$ g plasmid DNA at the lowest dose of $0.0002 \mu \mathrm{g} / \mathrm{ml} \mathrm{pFL}$-CMV. Increasing the dose to $2 \mu \mathrm{g} / \mathrm{ml}$ significantly reduced the value to 5,000 $\mathrm{pg} /$ well/ $\mu \mathrm{g}$ plasmid DNA. 


\section{Luciferase expression in B16-BL6 cells transfected with two different plasmids.}

Fig. 2 shows the amounts of firefly (Fig. 2A) and renilla luciferase (Fig. 2B) in cells 24 hr after cotransfection with $1.8 \mu \mathrm{g} / \mathrm{ml}$ pFL-TATA, pFL-SV40 or pFL-CMV and $0.2 \mu \mathrm{g} / \mathrm{ml} \mathrm{pRL}-\mathrm{TK}$, pRL-SV40 or pRL-CMV. The amount of firefly luciferase was the highest in cells transfected with pFL-CMV, which was about 4- and 20-fold higher than that in cells transfected with pFL-SV40 or pFL-TATA, respectively. In all cases examined, the amount of firefly luciferase was hardly affected by the cotransfection with renilla-expressing plasmid DNA (Fig. 2A). This was probably because the dose of the renilla luciferase-expressing plasmid DNA was only a tenth of the total amount of plasmids used for transfection. In marked contrast, the renilla luciferase activity in B16-BL6 cells was affected not only by the type of renilla luciferase-expressing plasmid DNA, but also by the type of competing, firefly luciferase expressing plasmid DNA (Fig. 2B). Renilla luciferase activity in cells transfected with pRL-CMV and pFL-CMV (pRL-CMV/pFL-CMV) or pRL-SV40/pFL-CMV was significantly lower than that in cells transfected with pRL-CMV/pFL-TATA or pRL-SV40/pFL-TATA, respectively. The amount of renilla luciferase in B16-BL6 cells transfected with pRL-TK was quite low compared with cells transfected with pRL-CMV or pRL-SV40.

Similar experiments were performed using $0.2 \mu \mathrm{g} / \mathrm{ml}$ firefly luciferase-expressing plasmid DNA and $1.8 \mu \mathrm{g} / \mathrm{ml}$ renilla luciferase-expressing plasmid DNA (Fig. 2C and D). The amount of firefly luciferase in cells cotransfected with pFL-CMV/pRL-CMV was significantly lower than 
that in cells cotransfected with pFL-CMV/pRL-TK (Fig. 2C). Again, the amount of renilla luciferase was hardly affected by the type of firefly luciferase expressing plasmid vectors cotransfected (Fig. 2D).

Fluorescent histogram of GFP in B16-BL6 cells transfected with pGFP-CMV and renilla luciferase-expressing plasmid.

Fig. 2E shows typical histograms of the fluorescence intensity of B16-BL6 cells transfected with pGFP-CMV and one of the following plasmids: pRL-CMV, pRL-SV40 or pRL-TK. In all cases examined, there was a large variation in the level of fluorescence intensity of B16-Bl6 cells transfected with pGFP-CMV. There were no obvious differences in the histograms of cells transfected with pGFP-CMV/pRL-TK or pGFP-CMV/pRL-SV40. On the other hand, the histogram of cells transfected with pGFP-CMV/pRL-CMV was different from the others; the percentage of cells with a high fluorescence intensity (the M1 region in the histogram) was $3 \%$, and this value was smaller than the 8 and $9 \%$ for the pGFP-CMV/pRL-SV40- and pGFP/pRL-TK-treated cells, respectively. These findings indicate that cotransfection of cells with pRL-CMV, the plasmid with the strongest promoter among those used, markedly reduced the expression of GFP from pGFP-CMV only in cells that received efficient delivery of these two plasmids. In accordance with these histograms, the mean fluorescence intensity of cells transfected with pGFP-CMV/pRL-CMV was significantly lower than that of cells transfected 
with pGFP-CMV/pRL-TK or pGFP-CMV/pRL-SV40 (Fig. 2F).

mRNA and protein expression level of luciferases in B16-BL6 cells transfected with two different plasmids.

The mRNA level was measured in B16-BL6 cells transfected with two types of plasmids, each of which expressed firefly or renilla luciferase. Again, cells were transfected with $0.2 \mu \mathrm{g} / \mathrm{ml}$ pFL-CMV and $1.8 \mu \mathrm{g} / \mathrm{ml}$ renilla luciferase-expressing plasmid, and the mRNA and luciferase activity were simultaneously measured at $24 \mathrm{hr}$ after transfection (Fig. 3). The amount of renilla luciferase protein was dependent on the type of plasmids used as described above, but it was almost proportional to its mRNA level in all cases (Fig. 3A). The amount of firefly luciferase protein was highly dependent on the type of renilla luciferase-expressing plasmid cotransfected (Fig. 3B), as shown in Fig. 2B. Moreover, the mRNA level of firefly luciferase was also affected by other plasmids in a manner dependent on the promoter strength (Fig. 3B), suggesting that some processes leading to mRNA expression are saturated in cells that produce a large amount of mRNA. In addition, the degree of reduction in firefly luciferase mRNA by cotransfection with pFL-CMV/pRL-CMV (about $20 \%$ of pFL-CMV/pRL-TK) was less than that of firefly luciferase protein (about $8 \%$ ), which implies that the amount of firefly luciferase protein was disproportionate to its mRNA level. 


\section{Luciferase expression in B16-BL6 cells transfected with siRNA and plasmids.}

The results obtained thus far are consistent with the hypothesis that the synthesis of protein from mRNA is saturated when a large amount of mRNA is transcribed, and this leads to disproportionate protein synthesis to the mRNA level. To confirm this hypothesis, we degraded the mRNA of renilla luciferase using siRL, an siRNA targeting renilla luciferase, and measured the amount of luciferase proteins. For this purpose, B16-BL6 cells were cotransfected with 1 $\mu \mathrm{g} / \mathrm{ml}$ siRL or siGFP, a control siRNA, in addition to $0.9 \mu \mathrm{g} / \mathrm{ml} \mathrm{pRL}-\mathrm{CMV}$, pRL-SV40 or pRL-TK and $0.1 \mu \mathrm{g} / \mathrm{ml} \mathrm{pFL}-\mathrm{CMV}$. As expected, the amount of renilla luciferase was markedly suppressed in cells transfected with siRL compared with siGFP (Fig. 4A), which suggests that siRL successfully degraded the target mRNA under these experimental conditions. Cotransfection of siRL significantly increased the amount of firefly luciferase in B16-BL6 cells transfected with pRL-CMV/pFL-CMV (Fig. 4B). These results suggest that siRNA-mediated degradation of mRNA from competing plasmids is effective in restoring transgene expression from the monitoring plasmid. However, the firefly luciferase expression in the cells transfected with siRL, pFL-CMV and pRL-CMV was lower than that of the cells transfected with siRL, pFL-CMV and pRL-TK.

Dose-dependent mRNA and protein expression of luciferase in mice after hydrodynamics-based administration and its effect on GAPDH expression. 
Fig. 5A shows the amounts of mRNA and protein of firefly luciferase in mouse liver 6 hr after hydrodynamic injection of pFL-CMV at different doses. Results were normalized to those of mice that received $1 \mu \mathrm{g} /$ mouse pFL-CMV administration. A linear correlation was observed between the firefly luciferase mRNA and the plasmid dose over the dose range investigated, i.e., from 1 to $300 \mu \mathrm{g} / \mathrm{mouse}$. At the dose of $10 \mu \mathrm{g} / \mathrm{mouse}$, the relative amount of luciferase protein was comparable with that of mRNA expression, indicating that the efficiency of protein synthesis from mRNA was similar at doses of 1 and $10 \mu \mathrm{g} /$ mouse. However, at a dose of $30 \mu \mathrm{g} / \mathrm{mouse}$ or higher, the ratio of firefly luciferase protein to its mRNA was smaller than at the low doses. Thus, these results indicate that the amount of luciferase protein becomes disproportionate to the amount of its mRNA in mouse liver, when the mRNA level is increased. Then, the mRNA and protein amounts of firefly luciferase were divided by the dose, and the values obtained were used to evaluate the efficiency or saturation of the expression. The efficiency of mRNA expression was not significantly affected by the dose, whereas that of protein expression significantly decreased with an increase in the dose of plasmid DNA.

To evaluate whether endogenous gene expression process is affected by large amount of mRNA expressed from plasmid vectors, the mRNA and protein expression of firefly luciferase and GAPDH in mouse liver were simultaneously measured $6 \mathrm{hr}$ after hydrodynamic administration of $0 \mu \mathrm{g}, 1 \mu \mathrm{g}$ or $100 \mu \mathrm{g}$ pFL-CMV (Fig. 5B). As is the case of the experiment above, the difference in mRNA amount of firefly luciferase between $1 \mu \mathrm{g}$ and $100 \mu \mathrm{g}$ was bigger 
than that in protein amount, which suggests the existence of saturation of protein synthesis process of transgene. No significant changes in GAPDH mRNA and protein expression were observed among all treatment group despite the fact that transgene expression process was saturated.

mRNA and protein expression of luciferases in mouse liver after hydrodynamic injection of naked plamid vectors.

Finally, mice received a hydrodynamic injection of $1 \mu \mathrm{g}$ pRL-CMV and $10 \mu \mathrm{g}$ pFL-CMV, pFL-SV40 or pFL-TATA, and the levels of mRNA and protein were simultaneously measured (Fig. 5C and D). The amount of firefly luciferase was dependent on the strength of promoter and proportional to its mRNA level (Fig. 5C). The mRNA level of renilla luciferase was almost the same in all cases (Fig. 5D), reflecting the fact that the same amount of pRL-CMV was used for hydrodynamic injection. In contrast, the amount of renilla luciferase protein in the liver of mice receiving $\mathrm{pRL}-\mathrm{CMV} / \mathrm{pFL-CMV}$ was significantly lower than that of mice receiving pRL-CMV/pFL-TATA (Fig. 5C).

\section{DISCUSSION}

Transgene expression from monitoring plasmid was reduced by cotransfection with competing plasmid driven by a strong promoter and this phenomenon was independent of the type of cDNA 
encoded in the monitoring and competing plasmids (Fig. 2). These results suggest that saturation of transgene expression is not dependent on the type of transgene product. In the experiment using pGFP-CMV, cells with a very high fluorescence intensity, which was found after cotransfection of cells with pRL-SV40 or pRL-TK, were not detected when cells were cotransfected with pRL-CMV. The cells with high fluorescence intensity should have been delivered with a large amount of pGFP-CMV and, therefore, they could have also efficiently taken up coexisting plasmids. Therefore, it is reasonable to suggest that transgene expression is saturated especially in cells that encounter a large amount of plasmid DNA. Although the both experiments exhibited a similar trend, the degree of the reduction by co-transfection with pRL-CMV was much less in the GFP experiment than that in the luciferase experiment. The discrepancy may be explained by the difference in the detection sensitivity of GFP and luciferase expression.

Transgene expression from plasmid DNA requires a number of processes, such as delivery of plasmid DNA to the nucleus, mRNA transcription from the DNA, export of mRNA from the nucleus, processing of mRNA, translation, and post-translational modification of protein. In the present study, we found that the level of mRNA and protein expression from monitoring plasmids was affected by the type of competing plasmids cotransfected in B16-BL6 cells (Fig. 3). Moreover, co-transfection of siRL with pFL-CMV (monitoring plasmid) and pRL-CMV (competing plasmid) significantly rescued firefly luciferase protein expression compared with 
the co-transfection of siGFP with pFL-CMV and pRL-CMV. These experimental results suggest for the first time that the translational process is highly likely to be saturated when a large amount of protein is produced. A careful comparison of the results showed that firefly luciferase expression in the cells transfected with pFL-CMV was lower when cells were cotransfected with siRL and pRL-CMV than that with siRL and pRL-TK. As renilla luciferase amount of the siRL/pFL-CMV/pRL-CMV group was much higher than that of the siRL/pFL-CMV/pRL-TK group, a simple explanation is that siRL-mediated reduction in renilla mRNA was not enough to completely rescue the firefly luciferase expression. We found a reduced mRNA expression of firefly luciferase in the pFL-CMV/pRL-CMV group compared to the pFL-CMV/pRL-TK group (Fig.3B). In addition, siRNA degrades its target mRNA in the cytoplasm so that siRNA does not affect the earlier processes such as transcription. Therefore, the siRL-mediated incomplete rescue of firefly luciferase protein expression may suggest that not only translation but also the earlier processes such as transcription are also saturated under the experimental conditions used.

The studies using mice led to a slightly different conclusion. Transgene expression was also saturated when a large amount of plasmid DNA was delivered, but the transcription was hardly affected by the dose (Fig. 5A). Therefore, the translational process is likely to be saturated in mouse liver (Fig. 5C and D), which is different from the in vitro results. This apparent discrepancy might be due to the difference in the type of transgene-expressing cells or in the delivery method of plasmid DNA. Further studies are needed to identify factors explaining the 
discrepancy. The firefly gene sequence in pFL-CMV was not fully codon-optimized for expression in mice and $5 \%$ of the codons in the sequence are ones with low frequency $(<30 \%)$ in mice, so this could be a bottleneck during translation. To our knowledge, no previous study has reported the saturation of transgene expression processes in vivo. Our finding agrees with the results of Carpentier et al (Carpentier et al. 2007). They found that transcriptional and translational processes are saturated under optimal transfection conditions in which cells were very efficiently transfected with plasmids. In addition, they reported that the translational process is saturated at a dose of plasmid DNA that does not affect the transcriptional process. In the present study, we found that transgene expression process can be saturated when the large amount of plasmid DNA is delivered to mouse liver by hydrodynamic injection. We and others also found a similar phenomenon in lipoplex-mediated and polyplex-mediated transfection of cultured cells, where the mode of gene delivery is different from the hydrodynamic injection (Carpentier et al. 2007). In addition, it has been reported that there is a saturation in secretory pathway after the transfection by adenoviral vectors encoding secretory proteins (Marmostein et al. 2000) Therefore, we believe that transgene expression could be saturated when cells are transfected using gene vectors with strong promoters.

When the transcription of endogenous genes is inhibited by adenovirus vectors because of the sharing of transcription factors, the expression of endogenous genes was inhibited at the mRNA level, and cells were damaged (Lin et al. 2007). As we observed saturation in the translational 
process, but not in the transcriptional process, this 'promoter squelching' will not be the case. Regarding toxicity, we did not observe any B16-BL6 cell death at $24 \mathrm{hr}$ after transfection. As an earlier study by Lin et.al reported that cellular toxicity by promoter squelching was observed at 48 and $72 \mathrm{hr}$ after transfection but not at $24 \mathrm{hr}$ after transfection, any toxicity in B16-BL6 cells might be observed at later time points. At least $6 \mathrm{hr}$ after the hydrodynamic delivery of plasmid DNA, no changes in the mRNA and protein amount of model endogenous gene, GAPDH, was observed. In addition, no damage was observed in the liver of mice at $6 \mathrm{hr}$ after hydrodynamic injection. In the case of hydrodynamic gene transfer, peak time of the transgene expression is usually about 6-24 hr and transgene expression level declines after the peak time. Therefore, transgene expression after hydrodynamic gene delivery is considered to be not long enough to affect endogenous gene expression.

In conclusion, we demonstrated that the transgene expression process can be saturated both in cultured cells and in mouse liver and that translation is a major process that can be saturated in transgene expression. Therefore, such an approach that can potentiate the gene expression processes of cells such as transcription and translation would be desirable to achieve higher gene expression if the delivery efficiency is high enough to saturate gene expression machinery.

\section{Acknowledgements}

This work was supported in part by Grants-in-Aid for Scientific Research from the Ministry of 
Education, Science, Sports, and Culture of Japan, by grants from the Ministry of Health, Labour and Welfare of Japan, and by a grant from the Program for Promotion of Fundamental Studies in Health Sciences of the National Institute of Biomedical Innovation (NIBIO).

\section{REFERENCES}

Carpentier E, Paris S, Kamen AA, Durocher Y. 2007. Limiting factors governing protein expression following polyethylenimine-mediated gene transfer in HEK293-EBNA1 cells. Journal of Biotechnology 128(2):268-280.

Cohen RN, van der Aa MAEM, Macaraeg N, Lee AP, Szoka Jr FC. 2009. Quantification of plasmid DNA copies in the nucleus after lipoplex and polyplex transfection. Journal of Controlled Release 135(2):166-174.

Dean DA. 1997. Import of plasmid DNA into the nucleus is sequence specific. Experimental Cell Research 230(2):293-302.

Dean DA, Dean BS, Muller S, Smith LC. 1999. Sequence requirements for plasmid nuclear import. Experimental Cell Research 253(2):713-722.

Herweijer H, Wolff JA. 2007. Gene therapy progress and prospects: Hydrodynamic gene delivery. Gene Therapy 14(2):99-107.

Kobayashi N, Nishikawa M, Hirata K, Takakura Y. 2004. Hydrodynamics-based procedure involves transient hyperpermeability in the hepatic cellular membrane: Implication of a 
nonspecific process in efficient intracellular gene delivery. Journal of Gene Medicine 6(5):584-592.

Kobayashi N, Nishikawa M, Takakura Y. 2005. The hydrodynamics-based procedure for controlling the pharmacokinetics of gene medicines at whole body, organ and cellular levels. Advanced Drug Delivery Reviews 57(5):713-731.

Lewis DL, Wolff JA. 2007. Systemic siRNA delivery via hydrodynamic intravascular injection. Advanced Drug Delivery Reviews 59(2-3):115-123.

Lin H, McGrath J, Wang P, Lee T. 2007. Cellular toxicity induced by SRF-mediated transcriptional squelching. Toxicological Sciences 96(1):83-91.

Liu F, Song YK, Liu D. 1999. Hydrodynamics-based transfection in animals by systemic administration of plasmid DNA. Gene Therapy 6(7):1258-1266.

Marmorstein AD, Csaky KG, Baffi J, Lam L, Rahaal F, Rodriguez-Boulan E. 2000. Saturation of, and competition for entry into, the apical secretory pathway. Proceedings of the National Academy of Sciences of the United States of America 97(7):3248-3253.

Miller AM, Dean DA. 2008. Cell-specific nuclear import of plasmid DNA in smooth muscle requires tissue-specific transcription factors and DNA sequences. Gene Therapy 15(15):1107-1115.

Nomura T, Yasuda K, Yamada T, Okamoto S, Mahato RI, Watanabe Y, Takakura Y, Hashida M. 1999. Gene expression and antitumor effects following direct interferon (IFN)- $\gamma$ gene transfer 
with naked plasmid DNA and DC-chol liposome complexes in mice. Gene Therapy 6(1):121-129.

Poste G, Doll J, Hart IR, Fidler IJ. 1980. In vitro selection of murine B16 melanoma variants with enhanced tissue-invasive properties. Cancer Research 40(5):1636-1644.

Tachibana R, Harashima H, Ide N, Ukitsu S, Ohta Y, Suzuki N, Kikuchi H, Shinohara Y, Kiwada H. 2002. Quantitative analysis of correlation between number of nuclear plasmids and gene expression activity after transfection with cationic liposomes. Pharmaceutical Research 19(4):377-381.

Takahashi Y, Nishikawa M, Kobayashi N, Takakura Y. 2005. Gene silencing in primary and metastatic tumors by small interfering RNA delivery in mice: Quantitative analysis using melanoma cells expressing firefly and sea pansy luciferases. Journal of Controlled Release 105(3):332-343.

FIGURE LEGENDS

Fig.1. Time- and dose-dependent luciferase expression in B16-BL6 cells after transfection of luciferase-expressing plasmid DNA. A. The firefly (closed symbols) and renilla (open symbols) luciferase activities were measured at indicated times after transfection of B16-BL6 cells with 1 $\mu \mathrm{g} / \mathrm{ml} \mathrm{pFL}-\mathrm{CMV}$ and $1 \mu \mathrm{g} / \mathrm{ml} \mathrm{pRL}-\mathrm{CMV}$. The results are expressed as the mean $\pm \mathrm{SD}(\mathrm{n}=4) . B$. 
The luciferase activity was measured at 1 day after transfection of B16-BL6 cells with the indicated dose of pFL-CMV (open triangle), pFL-SV40 (open circle) or pFL-TATA (closed circle). The results are expressed as the mean $\pm \mathrm{SD}(\mathrm{n}=3) . C$. The amount of firefly luciferase protein in Fig. $1 B$ was divided by the dose of plasmid DNA. The results are expressed as the mean \pm SD of the relative value to that of $0.0002 \mu \mathrm{g} / \mathrm{ml}(\mathrm{pFL}-\mathrm{CMV}$ and $\mathrm{pFL}-\mathrm{SV} 40)$ or to that of $0.002 \mu \mathrm{g} / \mathrm{ml}(\mathrm{pFL}-\mathrm{TATA})(\mathrm{n}=3)$.

Fig.2. Effect of the level of transgene expression from competing plasmid DNA on transgene expression from monitoring plasmid DNA. A \& B. B16-BL6 cells were cotransfected with $1.8 \mu \mathrm{g} / \mathrm{ml}$ pFL-TATA (closed bar), pFL-SV40 (open bar) or pFL-CMV (grey bar) and $0.2 \mu \mathrm{g} / \mathrm{ml} \mathrm{pRL}-\mathrm{TK}$ (closed bar), pRL-SV40 (open bar) or pRL-CMV (grey bar). One day after transfection, firefly (A) and renilla (B) luciferase activities were simultaneously measured. The results are expressed as the mean $\pm \mathrm{SD}(\mathrm{n}=4) . * \mathrm{P}<0.05$, compared with the pFL-TATA-transfected group. $C \& D$. B16-BL6 cells were cotransfected with $1.8 \mu \mathrm{g} / \mathrm{ml} \mathrm{pRL}$-TK (closed bar), pRL-SV40 (open bar) or pRL-CMV (grey bar) and $0.2 \mu \mathrm{g} / \mathrm{ml} \mathrm{pFL-TATA}$ (closed bar), pFL-SV40 (open bar) or pFL-CMV (grey bar). One day after transfection, firefly (C) and renilla (D) luciferase activities were simultaneously measured. The results are expressed as the mean $\pm \mathrm{SD}(\mathrm{n}=4) .{ }^{*} \mathrm{P}<0.05$ (vs pRL-TK transfected group). $E \& F$ B16-BL6 cells were cotransfected with $1.8 \mu \mathrm{g} / \mathrm{ml}$ pRL-CMV (line with shade), pRL-SV40 (bold line) or pRL-TK 
(solid line) and $0.2 \mu \mathrm{g} / \mathrm{ml} \mathrm{pGFP}-\mathrm{CMV}$. One day after transfection, the fluorescence intensity histogram (E) and mean fluorescence intensity (F) of cells were analyzed by flow cytometry. The results are expressed as the mean $\pm \mathrm{SD}(\mathrm{n}=4) . * \mathrm{P}<0.05$.

Fig.3. Effect of cotransfection on the levels of mRNA and protein in B16-BL6 cells. $A \& B$. B16-BL6 cells were cotransfected with $1.8 \mu \mathrm{g} / \mathrm{ml}$ pRL-TK (closed bar), pRL-SV40 (open bar) or pRL-CMV (grey bar) and $0.2 \mu \mathrm{g} / \mathrm{ml}$ pFL-CMV. One day after transfection, mRNA and protein expression levels of renilla (A) and firefly (B) luciferase were simultaneously measured. The results are expressed as the mean $\pm \mathrm{SD}(\mathrm{n}=4) . * \mathrm{P}<0.05$.

Fig.4. Effect of siRNA-mediated degradation of mRNA expressed from the competing plasmid on the expression from the monitoring plasmid. $A \& B$. B16-BL6 cells were cotransfected with siGFP (closed bar) or siRL (open bar) $(1 \mu \mathrm{g} / \mathrm{ml}), 0.9 \mu \mathrm{g} / \mathrm{ml} \mathrm{pRL}-\mathrm{CMV}$, pRL-SV40 or pRL-TK and $0.1 \mu \mathrm{g} / \mathrm{ml} \mathrm{pFL-CMV}$. One day after transfection, renilla (A) and firefly (B) luciferase activities were simultaneously measured. The results are expressed as the mean $\pm \mathrm{SD}(\mathrm{n}=4) . * \mathrm{P}<0.05$.

Fig.5. Effect of level of transgene expression from competing plasmid DNA on the expression from monitoring plasmid DNA and endogenous gene in mouse liver. A. Mice 
received a hydrodynamic injection of pFL-CMV at indicated doses. At 6 hr after injection, the level of mRNA (open triangle) and protein (closed circle) expression of firefly luciferase were simultaneously measured. The results are expressed as the mean $+\mathrm{SD}(\mathrm{n}=4) . B$. The amount of mRNA or protein expression in Fig. $5 A$ was divided by the dose. The results are expressed as the mean $+\mathrm{SD}$ of the relative value to that of $1 \mu \mathrm{g}$ group $(\mathrm{n}=4) .{ }^{*} \mathrm{P}<0.05$ vs $1 \mu \mathrm{g}$ group. $C$. Mice

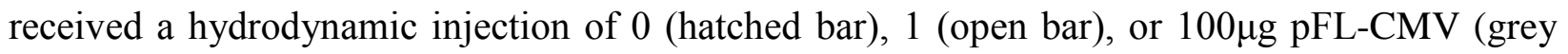
bar) or were untreated (closed bar). At $6 \mathrm{hr}$ after injection, the level of mRNA and protein expression of firefly luciferase and GAPDH were simultaneously measured. The results are expressed as mean \pm SD of the relative value to that of GAPDH of the no treatment groups $(\mathrm{n}=$ 4). $D \& E$. Mice received a hydrodynamic injection of $1 \mu \mathrm{g}$ pRL-CMV and $10 \mu \mathrm{g}$ pFL-TATA (closed bar), pFL-SV40 (open bar) or pFL-CMV (grey bar). At 6 hr after injection, mRNA and protein expression of firefly (C) and renilla (D) luciferase were simultaneously measured. The results are expressed as the mean $\pm S D(n=4) . * P<0.05$. 
Figure2

A

Monitoring plasmid Renilla

Competing plasmid Firefly
Monitoring plasmid Firefly

Competing plasmid Renilla GFP

Competing plasmid Renilla

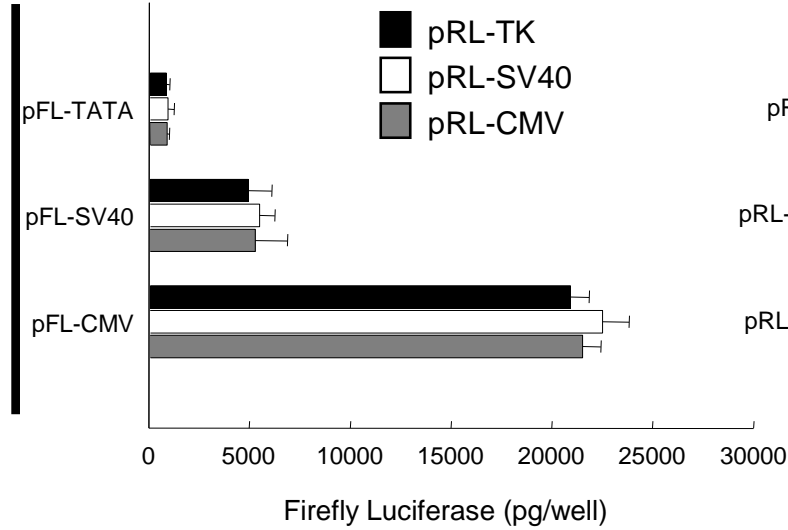

C

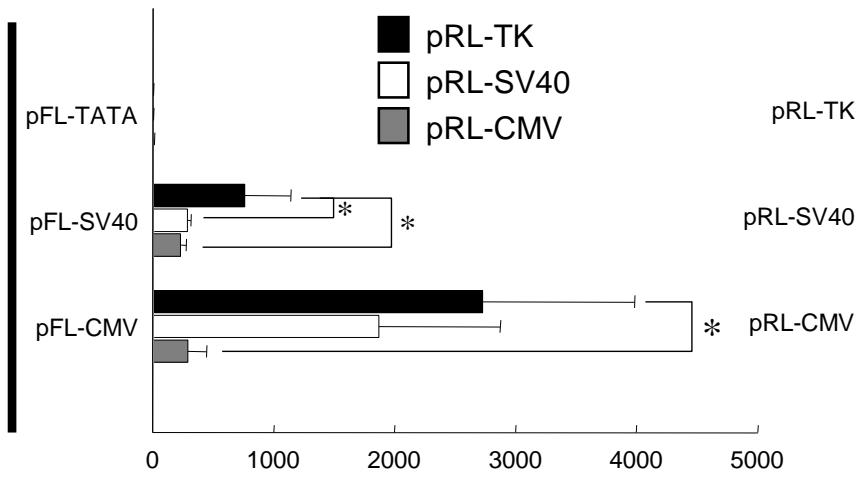

E

Firefly luciferase (pg/well)

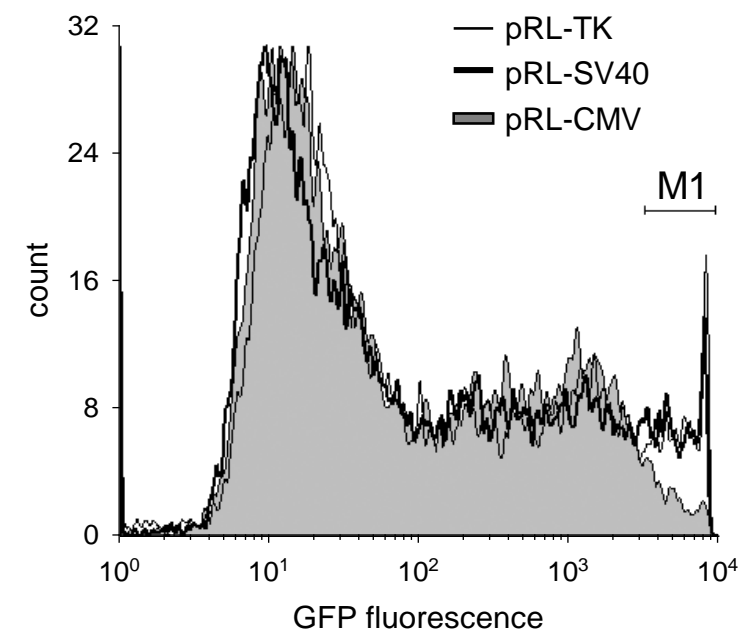

B

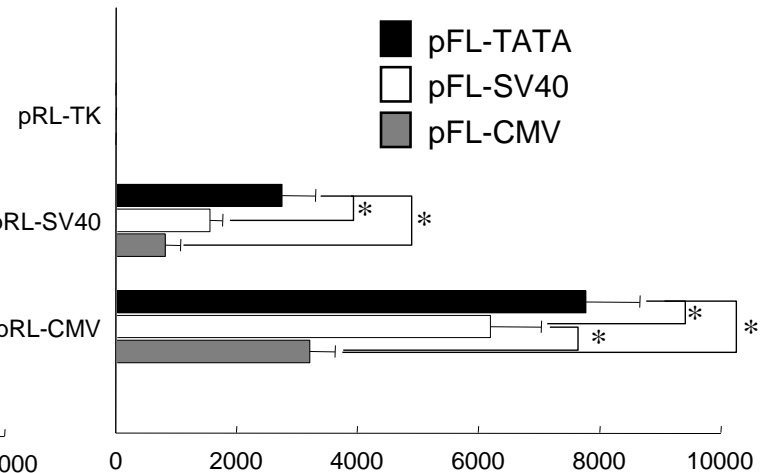

Renilla luciferase (pg/well)

\section{F}

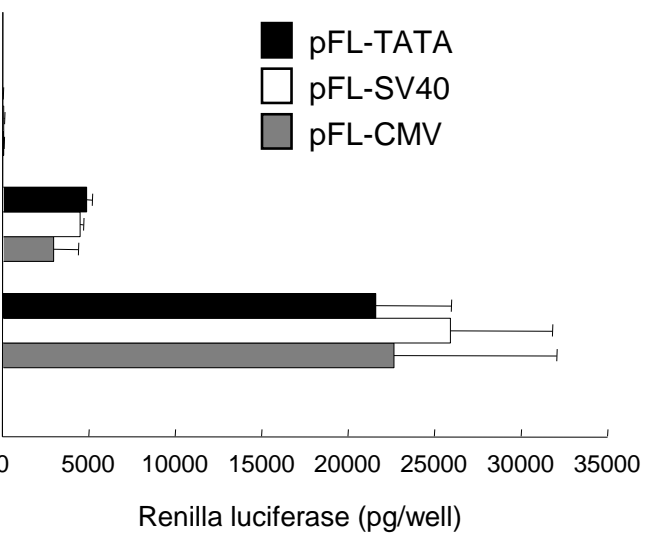

Monitoring plasmid 


\section{Figure4}

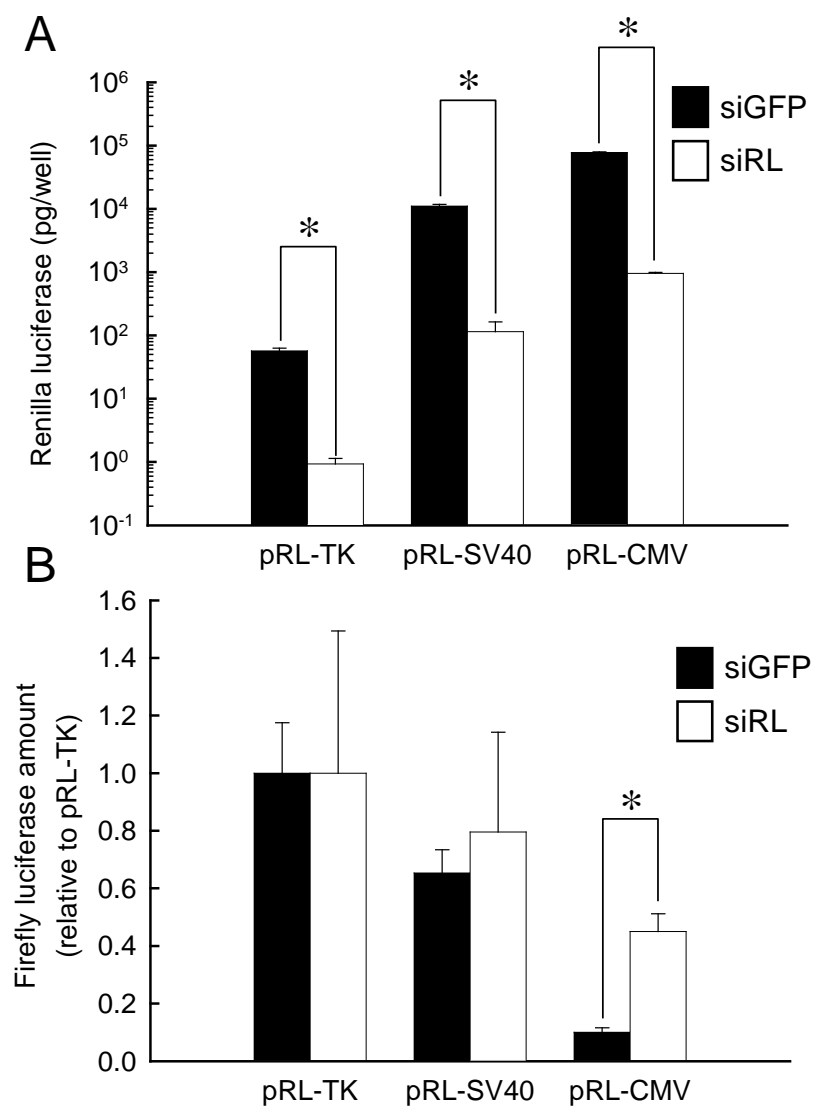


A

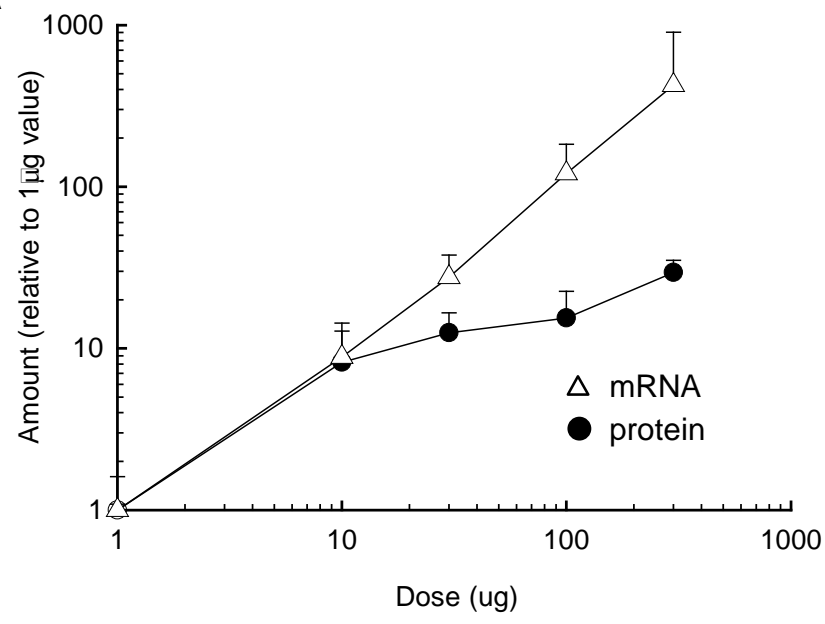

B

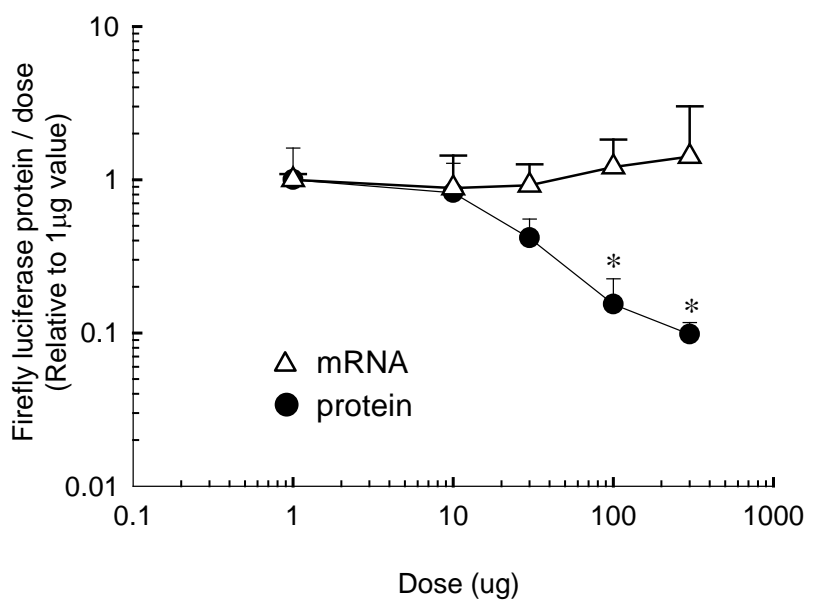

C

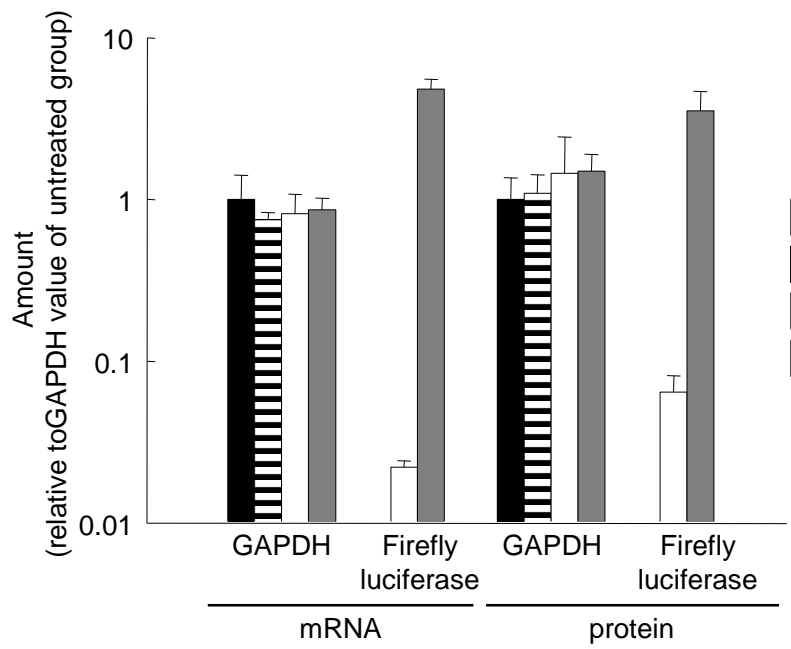

No Treat

目 Sham

$\square \mathrm{pFL}-\mathrm{CMV} 1 \mu \mathrm{g}$

pFL-CMV $100 \mu \mathrm{g}$

D

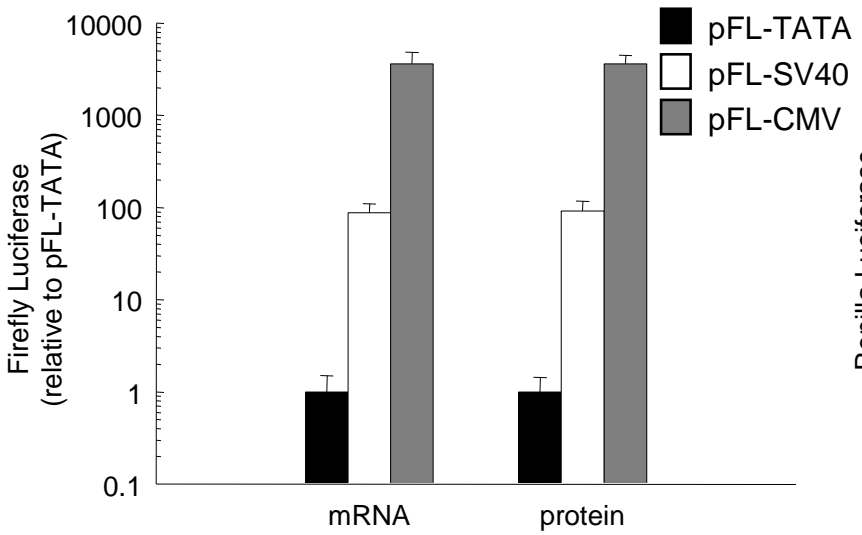

$E$

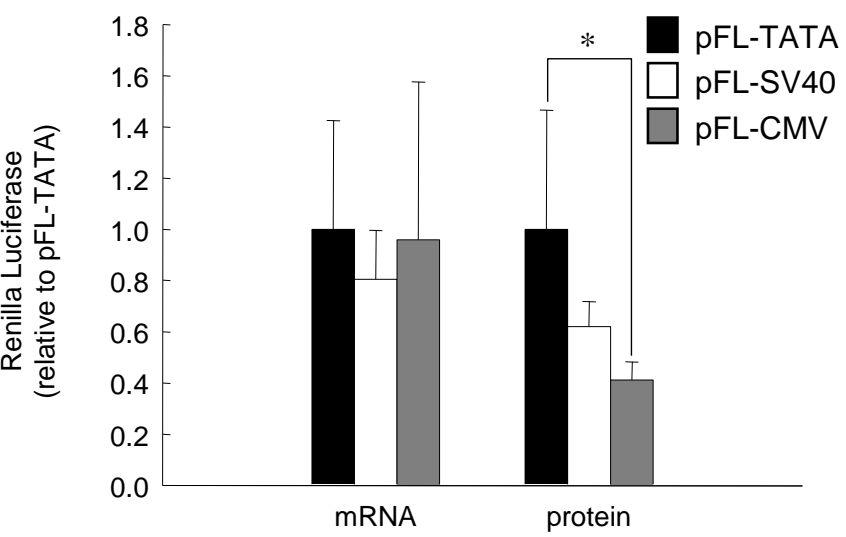

